\title{
Pressure-induced interlinking of carbon nanotubes
}

\author{
T. Yildirim, ${ }^{1}$ O. Gülseren, ${ }^{1,2}$ Ç. Kılıç, ${ }^{3}$ and S. Ciraci ${ }^{3}$ \\ ${ }^{1}$ NIST Center for Neutron Research, National Institute of Standards and Technology, Gaithersburg, Maryland 20899 \\ ${ }^{2}$ Department of Materials Science, University of Pennsylvania, Philadelphia, Pennsylvania 19104 \\ ${ }^{3}$ Physics Department, Bilkent University, Ankara 06533, Turkey
}

(Received 1 August 2000)

\begin{abstract}
We predict new forms of carbon consisting of one- and two-dimensional networks of interlinked single-wall carbon nanotubes, some of which are energetically more stable than van der Waals packing of the nanotubes on a hexagonal lattice. These interlinked nanotubes are further transformed with higher applied external pressures to more dense and complicated stable structures, in which curvature-induced carbon $s p^{3}$ rehybridizations are formed. We also discuss the energetics of the bond formation between nanotubes and the electronic properties of these predicted novel structures.
\end{abstract}

Carbon nanotubes, originally discovered as by-products of fullerene synthesis, ${ }^{1,2}$ are now considered to be the building blocks of future nanoscale electronic and mechanical devices. It is therefore desirable to have a good understanding of their electronic and mechanical properties and the interrelations between them. In particular, single-wall carbon nanotubes (SWNT's) provide a system where the electronic properties can be controlled by the structure of the nanotubes and by various deformations of their geometries. ${ }^{3-5}$ The physical properties can also be altered by intertube interactions between nanotubes packed in hexagonal lattices, as so called " "nanoropes."

The intertube interactions in nanoropes can be probed by applying external pressure to vary the intertube distance. ${ }^{6-8}$ For fullerenes, such high-pressure studies have yielded many interesting results including new compounds such as the pressure-induced polymeric phases of $\mathrm{C}_{60} \cdot{ }^{9}$ It is, therefore, of interest to inquire if similar covalent bonding can occur between the nanotubes in a rope. This could have important consequences for nanoscale device applications and composite materials that require strong mechanical properties since nanoropes consisting of interlinked SWNT will be significantly stronger than nanoropes composed of van der Waals packed nanotubes. ${ }^{10}$

A recent Raman study on SWNT ropes carried out up to 25.9 GPa (Ref. 7) showed that the mode intensities and energies are not completely reversible upon pressure cycling, suggesting irreversible pressure-induced changes in the structure. In another high-pressure study Chesnokov et al. ${ }^{8}$ observed a very large volume reduction and high compressibility, signaling the presence of a microscopic volumereducing deformation other than van der Waals compression. Some of these pressure-induced effects are tentatively attrib- uted to possible crushing or flattening the nanotube cross section from circular to elliptical or hexagonal. ${ }^{8}$ Motivated by these reports, we investigated possible new pressureinduced ground-state structures for $(n, 0)$ nanotube ropes ${ }^{11}$ from first-principles total energy calculations using the pseudopotential method within the generalized gradient approximation $^{12}$ (GGA). We observed an elliptical distortion of the nanotubes under pressure and subsequent curvatureinduced carbon rehybridization, giving rise to one- or twodimensional interlinked networks of nanotubes. This is somewhat reminiscent to the pressure-induced polymerizations of $\mathrm{C}_{60} \cdot{ }^{9}$

The first-principles total energy and electronic structure calculations were carried out using the pseudopotential plane wave code CASTEP. ${ }^{13}$ We used plane waves with an energy cutoff of $500 \mathrm{eV}$. With this cutoff and using ultra soft pseudopotentials for carbon, ${ }^{14}$ the total energy converges within $0.5 \mathrm{meV} /$ atom. For the Brillouin zone integration, we used between $3 \times 3 \times 6$ to $5 \times 5 \times 7 \mathrm{k}$ points according to the Monkhorst-Pack special k-point scheme. ${ }^{15}$ This method has already been applied to many carbon systems, including fullerenes and cubane with remarkable success. ${ }^{16}$

In this report, we present calculations on nanoropes consisting of $(5,0),(6,0),(7,0),(9,0)$, and $(6,6)$ nanotubes. For simplicity, we model the nanoropes as a hexagonal lattice of nanotubes. We further assume that we have only one nanotube per unit cell. $(6,0)$ nanotubes are perfectly compatible with the hexagonal lattice (i.e., $\gamma=120^{\circ}$ ). However in the optimized structure, hexagonal carbon rings of one nanotube face hexagonal rings from the neighboring tubes [Fig. 1(a)]. From studies of fullerenes, we know that the energy can be reduced by rotating every other tube such that their $\mathrm{C}-\mathrm{C}$ bonds face the center of the hexagonal faces of the neighbor-

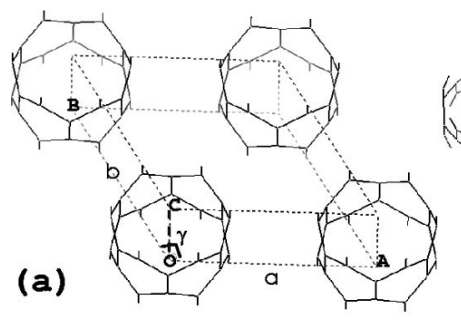

(b)

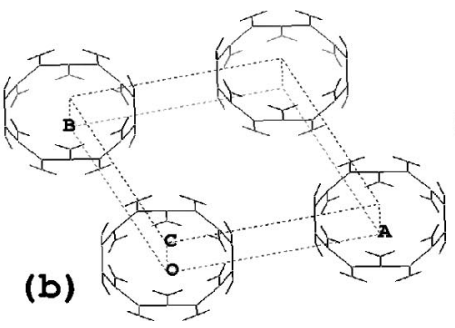

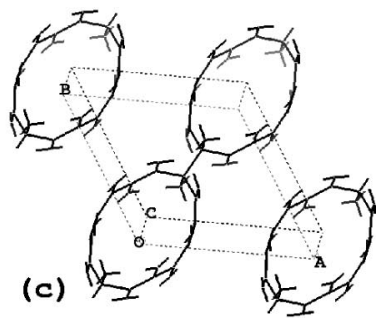

FIG. 1. Optimized structures of the vdW (a) $(6,0),(b)(7,0)$, and one-dimensional interlinked (c) $(7,0)$ nanotube lattices. The interlinked structure shown in (c) has lower energy than vdW packed $(7,0)$ nanotubes shown in $(b)$. 
TABLE I. Various structural parameters and the total energies of the optimized structures of $(n, 0)$ vdW lattices and one-dimensional interlinked (1DI) nanoropes as shown in Fig. 1. The band gaps of all the structures listed here are found to be zero, indicating metallic behavior.

\begin{tabular}{ccccccccc}
\hline \hline Properties & $(5,0)$ & $(5,0)(1 \mathrm{DI})$ & $(6,0)$ & $(6,0)(1 \mathrm{DI})$ & $(7,0)$ & $(7,0)(1 \mathrm{DI})$ & $(9,0)$ & $(9,0)(1 \mathrm{DI})$ \\
\hline Formula & $\mathrm{C}_{20}$ & $\mathrm{C}_{20}$ & $\mathrm{C}_{24}$ & $\mathrm{C}_{24}$ & $\mathrm{C}_{28}$ & $\mathrm{C}_{28}$ & $\mathrm{C}_{36}$ & $\mathrm{C}_{36}$ \\
Space group & $C m c m$ & $C m c m$ & $P 6 / \mathrm{mmm}$ & $\mathrm{Cmcm}$ & $\mathrm{Cmcm}$ & $\mathrm{Cmcm}$ & $P 63 / m c m$ & $\mathrm{Cmcm}$ \\
$a=b(\AA)$ & 7.408 & 7.079 & 8.364 & 7.762 & 9.250 & 8.432 & 10.389 & 9.532 \\
$c(\AA)$ & 4.208 & 4.190 & 4.212 & 4.223 & 4.218 & 4.205 & 4.219 & 4.209 \\
$\gamma$ & 72.46 & 125.39 & 120.00 & 121.96 & 102.35 & 119.15 & 120.00 & 111.97 \\
Density $\left(\mathrm{g} / \mathrm{cm}^{3}\right)$ & 1.8119 & 2.331 & 1.8713 & 2.2176 & 1.5849 & 2.1415 & 1.8213 & 2.0245 \\
Energy/C $(\mathrm{eV})$ & -155.694 & -155.802 & -155.843 & -155.868 & -155.946 & -155.969 & -156.049 & -156.042 \\
\hline \hline
\end{tabular}

ing nanotubes. This would double the unit cell for $(6,0)$ nanotubes. We will not pursue this here because we are mainly interested in the potential for covalent bonding between nanotubes.

$(9,0)$ nanotubes are also compatible with hexagonal symmetry. Furthermore the relative orientation of the nanotubes are optimal energetically (i.e., $\mathrm{C}-\mathrm{C}$ bonds face the center of the hexagonal faces of adjacent nanotubes). $(5,0)$ and $(7,0)$ nanotubes are not compatible with hexagonal symmetry and therefore $\gamma$ is expected to deviate from the ideal value of $120^{\circ}$. We find that when one bond is along the $a$ axis, the total energy is minimized when the $b$ axis is aligned so that it brings the $\mathrm{C}-\mathrm{C}$ bonds of one nanotube to the center of hexagonal faces of adjacent nanotubes. For $(5,0)$ and $(7,0)$ nanotubes this occurs when $\gamma=(360 / 5) \times 1=72.0^{\circ}$ and $(360 / 7) \times 2=102.857^{\circ}$, respectively. These values are very close to the values $\gamma=72.46^{\circ}$ and $102.35^{\circ}$ obtained from the first-principles structural optimization.

The pressure dependence of these lattices of nanotubes was determined by calculating the total energy as a function of nanotube separation (i.e., $a$ and $b$ ) while the other parameters, including atom positions, $c$, and $\gamma$ are optimized. We observe that $(7,0)$ nanotubes become elliptically distorted with applied pressure (i.e., decreasing nanotube-nanotube distance). At a critical pressure, we observe a structural phase transformation from the van der Waals nanotube lattice [as shown in Figs. 1(a) and 1(b)] to a new lattice in which the nanotubes are interlinked along the [110] direction, where the strain of the nanotube is largest [Fig. 1(c)]. The covalent bonding between nanotubes is therefore the result of curvature-induced rehybridization of the carbon orbitals. The same structural transformation was observed for the other $(n, 0)$ nanoropes and the results are summarized in Table I. The structure of each of these one-dimensionally interlinked nanoropes is orthorhombic (space group $\mathrm{Cmcm}$ ), with two nanotubes per unit cell. The relationship between the conventional orthorhombic cell and the primitive one is shown in Fig. 2(a).

Figure 2 also shows the local environment of the carbon atoms involved in the interbonding of the nanotubes for $(6,0)$ and $(7,0)$. In the first case, the covalent bonding occurs between carbon atoms on two hexagonal rings [Fig. 2(b)]. We believe this process is slightly less favorable than covalent bonding between carbons on a hexagonal ring and those involved in an intratube $\mathrm{C}-\mathrm{C}$ bond which occurs for the $(7,0)$ case [Fig. 2(c)]. In both cases, the bond distances are com- parable to those in diamond, indicating $s p^{3}$ hybridization. The bond angles vary from about $100^{\circ}$ to $120^{\circ}$, indicating some strain. From Table I, we see that the energy of the interlinked phase is actually lower than the van der Waals $(\mathrm{vdW})$ lattices for $(5,0)$ and $(7,0)$ nanotropes. For the $(9,0)$ nanorope, the energy difference is relatively small. Regardless of the energies relative to that of unlinked nanotubes, an interlinked phase is stable once it is formed, because breaking the intertube bonds requires jumping over a significant energy barrier.

To quantitatively study the bonding mechanism, we calculated the total energies of the different phases as a function of the lattice constant (i.e., applied pressure). The result for $(7,0)$ nanotubes is summarized in Fig. 3. The energies of the $\mathrm{vdW}$ and the one-dimensional interlinked (1DI) phases cross

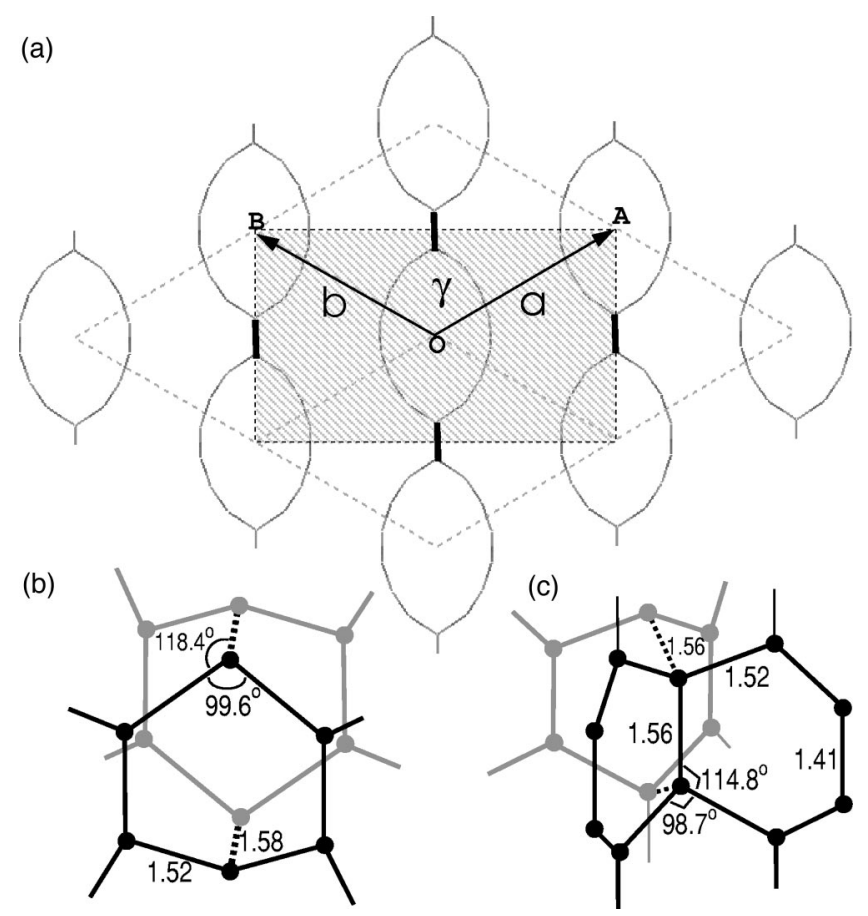

FIG. 2. (a) A view along the $c$ axis of the $1 \mathrm{D}$ interlinked $(n, 0)$ nanotube lattice. The shaded rectangular region is the orthorhombic unit cell. Local structure of carbon atoms involved in the intertube bonding (dotted lines) between two (b) $(6,0)$ and (c) $(7,0)$ nanotubes. The $s p^{3}$ hybridization occurs between two hexagonal faces for $(6,0)$ nanotubes and between a hexagonal face and a $\mathrm{C}-\mathrm{C}$ bond for $(7,0)$ nanotubes. 


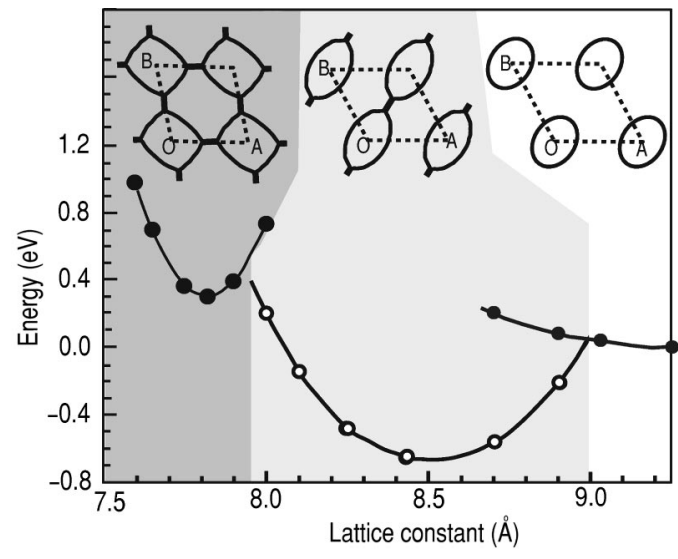

FIG. 3. Planar lattice constant variation of the total energy of $(7,0)$ nanotube ropes in three different phases. Inset shows the view of the structures along the $c$ axis. The zero of energy was taken to be the energy of vdW packing of the nanotubes.

each other at about $a=9.0 \AA$ with an energy barrier of only $46 \mathrm{meV} /$ unitcell $(552 \mathrm{~K})$. The pressure required to attain this lattice constant is only about $0.3 \mathrm{GPa}$ for the vdW phase, indicating that polymerization of $\mathrm{vdW}(7,0)$ nanoropes could occur at modest pressures and temperatures. ${ }^{17}$ Once the interlinked phase is reached, the energy barrier required to break the bonds and obtain free nanotubes is about $0.7 \mathrm{eV}$ ( $25 \mathrm{meV} /$ atom), which is comparable to that of $1 \mathrm{D}$ polymerized $\mathrm{C}_{60}$ molecules $(20 \mathrm{meV} /$ atom $) .{ }^{18}$

Figure 3 also shows that another interlinked phase of $(7,0)$ nanotubes becomes the ground state for lattice parameter smaller than $8.0 \AA$. In this new phase the nanotubes are interlinked along both $a$ and $b$ axes [see Fig. 4(a)]. This twodimensional interlinked (2DI) structure is about four times stiffer (i.e., $d^{2} E / d a^{2}=13.7 \mathrm{eV} / \AA^{2}$ ) than the 1D interlinked phase $\left(d^{2} E / d a^{2}=3.3 \mathrm{eV} / \AA^{2}\right)$ and sixteen times stiffer than the vdW nanoropes $\left(d^{2} E / d a^{2}=0.8 \mathrm{eV} / \AA^{2}\right)$.

We observe that applying even higher pressures yields more complicated and denser phases for many of the nanoropes studied here (see Fig. 4). For $(9,0)$ nanoropes, we find that the nanotubes are interlinked along three directions forming a hexagonal network. The length of the intertube bond, $d_{\mathrm{C}-\mathrm{C}}=1.644 \AA$, is significantly elongated for an $s p^{3}$ $\mathrm{C}-\mathrm{C}$ bond. The two-dimensional interlinked phase of $(7,0)$ nanotubes is further transformed to a denser structure at 30 GPa [Fig. 4(c)]. By comparison, $(6,6)$ nanotubes do not form an interlinked structure up to a pressure of $60 \mathrm{GPa}$. Rather the nanotubes are hexagonally distorted such that the local structure of the nanotube faces is similar to that in graphite sheets [Fig. 4(c)]. Furthermore, releasing the pressure yields the original structure, indicating that the distortion is purely elastic. Similar calculations are currently underway for other $(n, m)$ nanotubes. However, we expect similar results for other types of tubes.

A detailed discussion of the electronic band structure and density of states of the predicted structures will be presented elsewhere, however all the structures reported here [except one in Fig. 4(c)] are found to be metal. The dispersion of the bands near Fermi level in a direction perpendicular to the tube axis is found to be around $0.5 \mathrm{eV}$, making the $\mathrm{vdW}$ nanoropes metallic even though the individual tubes are insulating [such as $(7,0)$ nanotubes] or semiconducting with a
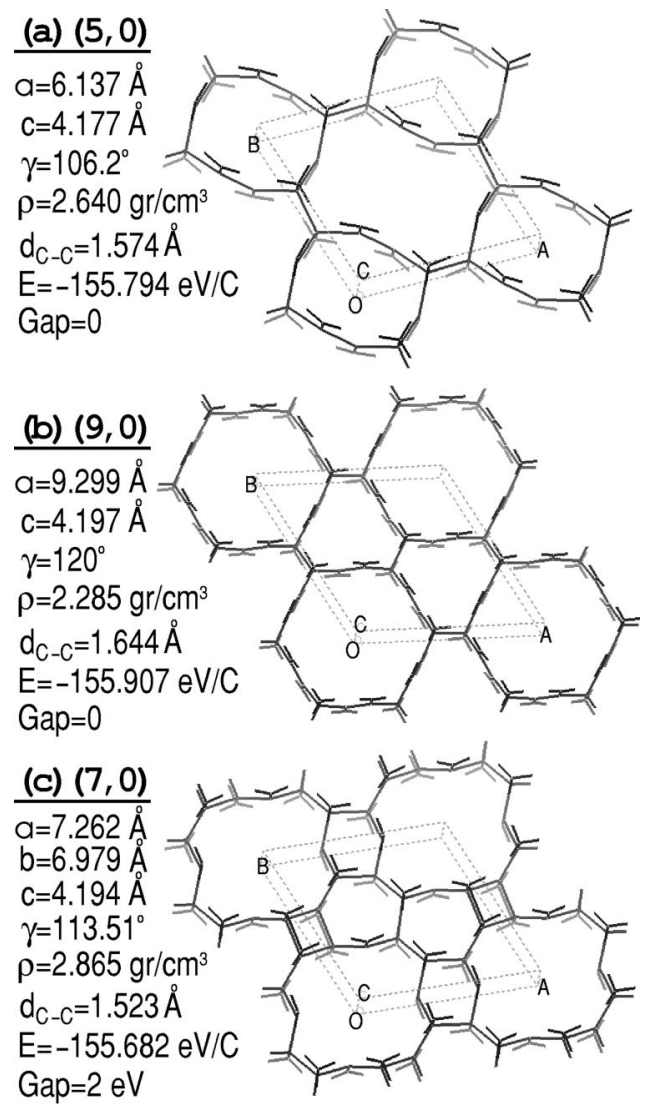

$\mathrm{Gap}=2 \mathrm{eV}$

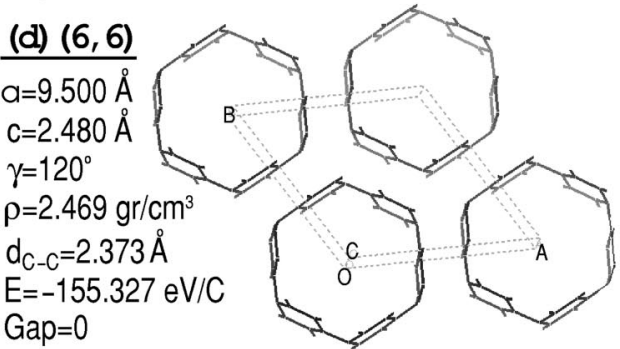

FIG. 4. Various high density phases of carbon nanotubes. (a) Two-dimensional interlinked (2DI) structure of $(5,0)$ nanotubes, consisting of rectangularly distorted nanotubes interlinked on a $2 \mathrm{D}$ network. (b) A hexagonal network of $(9,0)$ nanotubes, in which $(9,0)$ nanotubes are interlinked along $a, b$, and [110] directions. (c) A very dense structure of $(7,0)$ nanotubes obtained under $30 \mathrm{GPa}$ pressure. (d) The optimized structure of $(6,6)$ nanotubes under $P$ $=53 \mathrm{GPa}$. Nanotubes are distorted in such a way that the local nearest-neighbor structure is somewhat similar to graphite sheets. $d_{\mathrm{C}-\mathrm{C}}$ indicates the smallest distance between two carbon atoms of nearest neighbor nanotubes.

small band gap. The structural changes clearly have strong effects on the electronic properties and therefore should be detected in the pressure dependence of various transport properties of nanoropes. From our calculations we expect two effects to be observed. First, on the initial pressure cycle one should observe an irreversible effect in the resistivity due to the polymerization of the nanotubes. Second, the applied pressure will induce a reversible deformation of the circular cross section, which will change the metallic behavior of the nanotubes. ${ }^{5}$ For example, the $(7,0)$ nanorope, which is found to be metallic at ambient pressure, transforms to a high-density phase as shown in Fig. 4(c) at $30 \mathrm{GPa}$, 
which is found to be a band insulator with a band gap of 2 eV.

The new pressure-induced, high-density phases reported here can also be important host lattices for intercalation and sorption. For instance, the high-density phase of $(7,0)$ nanoropes [Fig. 4(c)] has an interesting structure in which some parts of the lattice are very strained containing $s p^{3}$ bonded square carbon rings, while other parts consist of graphitelike $s p^{2}$ bonded carbon atoms (28\% of all atoms). In this structure, there are still interstitial sites that would accommodate other species such as alkali metals.

In summary, we have presented first-principles calculations of the structures and electronic properties of various nanoropes. We find that small nanotubes in a rope are distorted elliptically with applied pressure and then are covalently bonded to each other at the positions of highest curvature point of nanotubes by $s p^{3}$ hybridization of the carbon orbitals. For small nanotubes, the resulting onedimensional chains of elliptic carbon nanotube structure are found to be energetically more stable than the circular van der Waals nanoropes. Higher applied pressures resulted in more dense and complicated structures. Thus pressure induced polymerization of the nanotubes may provide a way of synthesizing novel carbon base materials with interesting physical properties. For example interlinking of the nanotubes may improve the mechanical performance of composites based on these materials. It will be an experimental challenge to confirm the structures predicted here. A difference NMR spectrum of two identical samples; one treated with pressure and the other not, may give some evidence for the new phases. Similar difference measurements by ESR and Raman could be equally valuable.

We thank D. A. Neumann, R. L. Cappelletti, and J. E. Fischer for many fruitful discussions and critical reading of the manuscript. This work is partially supported by the National Science Foundation under Grant No. INT97-31014 and TÜBITAK under Grant No. TBAG-1668(197 T 116).
${ }^{1}$ S. Iijima, Nature (London) 354, 56 (1991).

${ }^{2}$ S. Iijima, T. Ichihashi, and Y. Ando, Nature (London) 356, 776 (1992).

${ }^{3}$ N. Hamada, S. Sawada, and A. Oshiyama, Phys. Rev. Lett. 68, 1579 (1992).

${ }^{4}$ C. J. Park, Y. H. Kim, and K. J. Chang, Phys. Rev. B 60, 10656 (1999).

${ }^{5}$ O. Gülseren et al. (unpublished).

${ }^{6}$ J. R. Wood, M. D. Frogley, E. R. Meurs, A. D. Prins, T. Peijs, D. J. Dunstan, and H. D. Wagner, J. Phys. Chem. B 103, 10388 (1999).

${ }^{7}$ U. D. Venkateswaran, A. M. Rao, E. Richter, M. Menon, A. Rinzler, R. E. Smalley, and P. C. Eklund, Phys. Rev. B 59, 10928 (1999); A. K. Sood, Pallavi V. Teredesai, D. V. S. Muthu, R. Sen, A. Govindaraj, and C. N. R. Rao, Chem. Phys. Lett. 319, 296 (2000); P. V. Teredesai, A. K. Sood, D. V. S. Muthu, R. Sen, A. Govindaraj, and C. N. R. Rao, Chem. Phys. Lett. 319, 296 (2000).

${ }^{8}$ S. A. Chesnokov, V. A. Nalimova, A. G. Rinzler, R. E. Smalley, and J. E. Fischer, Phys. Rev. Lett. 82, 343 (1999).

${ }^{9}$ A. M. Rao et al., Appl. Phys. A: Mater. Sci. Process. 64, 231 (1997).

${ }^{10}$ J. P. Salvetat et al., Phys. Rev. Lett. 82, 944 (1999).

${ }^{11}$ Structure of a nanotube is described by two integers $(n, m)$ as explained by M. S. Dresselhaus, G. Dresselhaus, and R. Saito, Phys. Rev. B 45, 6234 (1992).

${ }^{12}$ J. P. Perdew and Y. Wang, Phys. Rev. B 46, 6671 (1992).

${ }^{13}$ M. C. Payne, M. P. Teter, D. C. Allen, T. A. Arias, and J. D. Joannopoulos, Rev. Mod. Phys. 64, 1045 (1992).

${ }^{14}$ D. Vanderbilt, Phys. Rev. B 41, 7892 (1990).

${ }^{15}$ H. J. Monkhorst and J. D. Pack, Phys. Rev. B 13, 5188 (1976).

${ }^{16}$ T. Yildirim, S. Ciraci, C. Kılıç, and A. Buldum, Phys. Rev. B 62, 7625 (2000).

${ }^{17}$ We note that the transition path should be calculated in order to obtain the actual barriers between the different phases, which is not done in this paper.

${ }^{18}$ G. B. Adams, J. B. Page, O. F. Sankey, and M. O'Keeffe, Phys. Rev. B 50, 17471 (1994). 